\title{
Simple model for the kinetics of packaging of DNA into a capsid against an external force
}

\author{
K. P. Santo and K. L. Sebastian \\ Department of Inorganic and Physical Chemistry, Indian Institute of Science, Bangalore 560012, India
}

\begin{abstract}
We propose a simple model for the kinetics of packaging of viral DNA into a capsid against an external force trying to prevent it. The model leads to a Butler-Volmer-type dependence of the rate of packaging on the pulling force $F$.
\end{abstract}

Recently, in a very interesting experiment [1], the rate of packaging of a long viral DNA into a capsid by means of the portal complex, under the influence of an external force trying to prevent the packaging has been studied (see Fig. 1). The force-velocity $(F-\nu)$ curves show that packaging rate decreases even for small forces, implying that the ratedetermining step in the packaging is affected by the externally applied force. Using a Kramer's-type model, involving steps that involve thermal activation over a barrier, the experimental data was fitted assuming two force-dependent steps. The first involves a conformational change of only 0.1 $\mathrm{nm}$ and is the rate-determining step for small forces. At higher forces, the velocity is found to decrease more sharply and it was suggested that the second step, which has a larger conformational change associated with it, becomes the ratelimiting step. In the following, we present a simple theoretical model for the $F-\nu$ curves, which seems to fit the data reasonably well. In our model, there is only one step in the activation process, and we include the possibility of the reverse process too. Interestingly the model leads to a formula analogous to the Butler-Volmer equation of electrochemistry. A mechanism consistent with the structure of the packaging motor, determined from x-ray crystallography has been suggested [2]. According to this, the packaging involves successive firing of five ATPases in the portal complex. The hydrolysis of one adenosine triphosphate (ATP) molecule leads to a movement of two base pairs of the DNA into the capsid. To analyze the process, using the methods of physical kinetics, we need to have an idea of of the potential-energy surface for the processes, and of the transition state. Here, it is most convenient to take the reaction coordinate to be the length of the DNA molecule inside the capsid, which we shall denote as $x$. Every increase in $x$ by $a(=6.8 \AA)$ involves the hydrolysis of one ATP molecule. This hydrolysis is coupled mechanically to the movement of the DNA, through the ATPase. Therefore, as $x$ changes by $a$, there is a lowering of net free energy of the system.

A free-energy profile for the process, against the reaction coordinate $(x)$ is shown in Fig. 2. Each minimum $(\mathrm{A}, \mathrm{B}, \mathrm{C})$ in the curve is an initial state for the packaging of two more base pairs, and has one ATP molecule, ready to be hydrolyzed. In going from A to B, the ATP is hydrolyzed to adenosine diphosphate (ADP) (leading to a lowering of free energy) and two base pairs have been packed in. At B, the ADP is got rid of, and another ATP is attached (not to the same ATPase, see Ref. [2]) and then the system goes over from $\mathrm{B}$ to $\mathrm{C}$. The experiments are done under saturating concentration of ATP. Hence, ATP attachment is not the ratedetermining step.

The transition state is at a distance of $\Delta x$ from A. To reach it in the presence of an external force $F$, an extra work of $F \Delta x$ is required. Therefore the rate of the forward process, in the presence of the force is given by $\nu_{0 f} \exp (-F \Delta x / k T)$, where $\nu_{0 f}$ is the rate in the absence of the external force. The rate of the backward reaction is, in a similar fashion, given by $\nu_{0 b} \exp [F(a-\Delta x) / k T]$, where $a$ $=6.8 \AA$. Hence, the net rate is $\nu=\nu_{0 f} \exp (-F a \alpha / k T)$ $-\nu_{0 b} \exp [F a(1-\alpha) / k T]$, where $\alpha=\Delta x / a$. Using this expression, we have fitted the $F-\nu$ curves in Ref. [1] and the results are shown in Fig. 3. The equation seems to fit the data fairly well. Reference [1] assumes two different ratedetermining steps, operating at different force strengths. Further, they have four unknown parameters which are used for fitting. In comparison, in our mechanism, there is only one (reversible step) and three parameters. Interestingly, the above equation is very similar to the Butler-Volmer equation of electrochemistry [3], in which the force $F$ plays a role similar to the overpotential, and $\alpha$ is the analog of the symmetry factor. We have used the equation to fit the data using MATHEMATICA. At $1 / 3$ packing the best fit is obtained for the parameters $\Delta x=0.769 \AA, \nu_{0 f}=100.754$ base pairs per second $(\mathrm{bp} / \mathrm{s})$ and $\nu_{0 b}=8.704 \times 10^{-3} \mathrm{bp} / \mathrm{s}$ with an error of $\pm 1.16 \mathrm{bp} / \mathrm{s}$. For $2 / 3$ packing, the best-fit parameters were $\Delta x=0.989 \AA, \quad \nu_{0 f}=82.859 \mathrm{bp} / \mathrm{s}, \quad$ and $\quad \nu_{0 b}=4.556$ $\times 10^{-2} \mathrm{bp} / \mathrm{s}$ with an error of $\pm 1.63 \mathrm{bp} / \mathrm{s}$. However, it seems best to interpret $\Delta x$ as perhaps a structural parameter, inde-

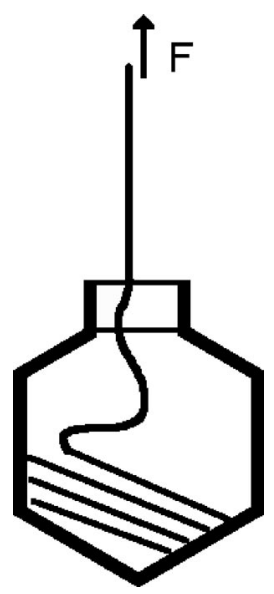

FIG. 1. Packaging of the DNA into the capsid against an external force $F$ trying to prevent it. 


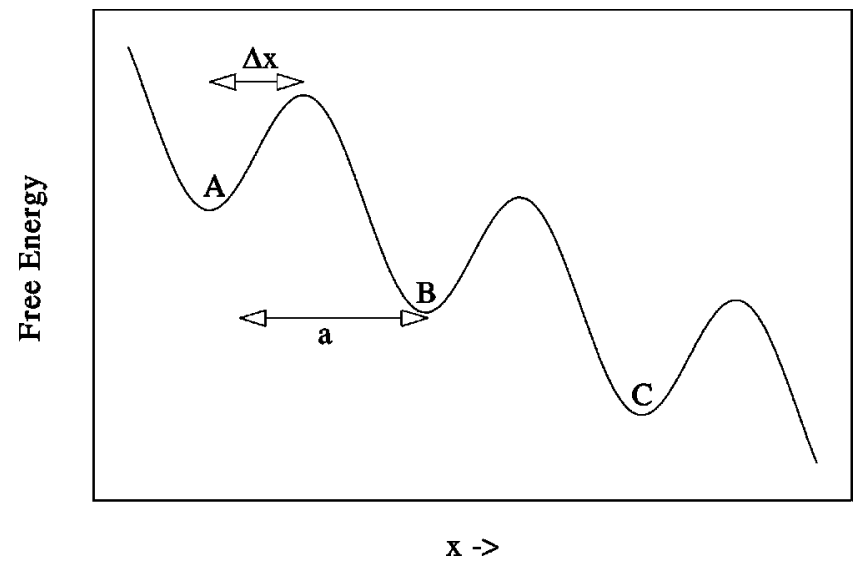

FIG. 2. Free-energy profile for the packaging. $x$ denotes the length of the DNA that has been packaged. The packaging of a length $a$ corresponds to going from A to $\mathrm{B}$ (or B to $\mathrm{C}$ ) and involves the hydrolysis of one ATP molecule. As more and more DNA gets packed in the oscillations in the free-energy profile would decrease and the profile would get flatter and flatter.

pendent of the amount of packing. Therefore, we fitted the two cases with the same value of $\Delta x$ and this lead to $\Delta x$ $=0.802 \AA$ and $\nu_{0 f}=101.980 \mathrm{bp} / \mathrm{s}$ and $\nu_{0 b}=8.469$ $\times 10^{-3} \mathrm{bp} / \mathrm{s}$ with an error of $\pm 1.30 \mathrm{bp} / \mathrm{s}$ for the $1 / 3$ packing and $\nu_{0 f}=77.753 \mathrm{bp} / \mathrm{s}$ and $\nu_{0 b}=4.850 \times 10^{-2} \mathrm{bp} / \mathrm{s}$ with an error of $\pm 2.52 \mathrm{bp} / \mathrm{s}$ for the $2 / 3$ packing. The results for this case are shown in the Fig. 3. We used this fitting to calculate

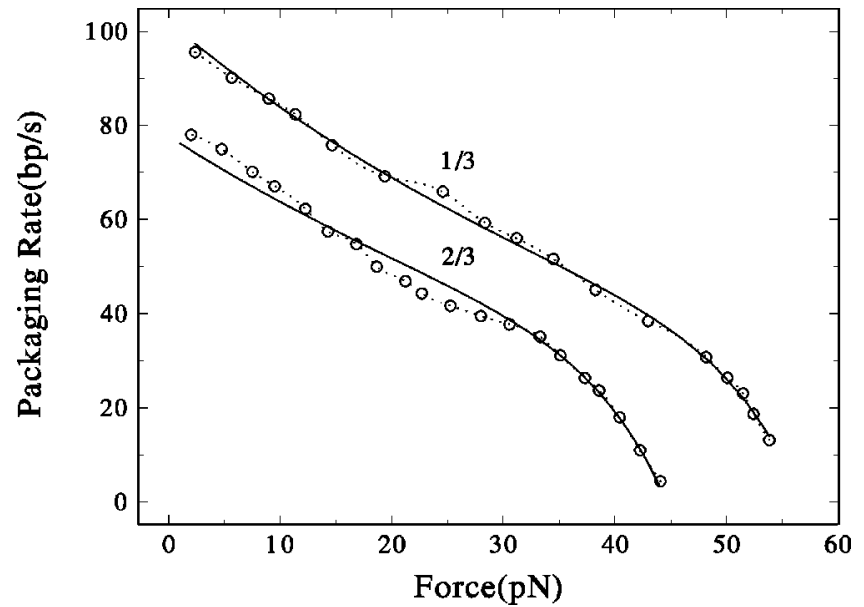

FIG. 3. Packaging rate $\nu$ against the force. The best fit was obtained using MATHEMATICA. A value of $\Delta x=0.802 \AA$ leads to the best results. At $1 / 3$ packing this we get $\nu_{0 f}=101.980$ base pairs per second $(\mathrm{bp} / \mathrm{s})$ and $\nu_{0 b}=8.469 \times 10^{-3} \mathrm{bp} / \mathrm{s}$ with an error of $\pm 1.30 \mathrm{bp} / \mathrm{s}$ and $\nu_{0 f}=77.753 \mathrm{bp} / \mathrm{s}$ and $\nu_{0 b}=4.850 \times 10^{-2} \mathrm{bp} / \mathrm{s}$ with an error of $\pm 2.52 \mathrm{bp} / \mathrm{s}$ for the $2 / 3$ packing.

the force at which the packaging rate would become zero and we find that the values are 56.85 and $44.65 \mathrm{pN}$, respectively, at the two packings. The difference is $12.2 \mathrm{pN}$, which is to be compared with the value of $14 p N$ of [1].

K.P. Santo is grateful to CSIR (India), for financial support.
[1] D.E. Smith, S.J. Tans, S.B. Smith, S. Grimes, D.L. Anderson, and C. Bustamante, Nature (London) 413, 748 (2001).

[2] A.A. Simpson, Y. Tao, P.G. Leiman, M.O. Badasso, Y. He, P.J. Jardine, N.H. Olson, M.C. Morals, S. Grimes, D.L. Anderson,
T.S. Baker, and M.G. Rossmann, Nature (London) 408, 745 (2000).

[3] P.W. Atkins, Physical Chemistry (Oxford University, New York, 1998), p. 883. 